\title{
Numerical Simulation of Dislocation Cross-Slip with Annihilation in Non-Symmetric Configuration
}

\author{
P. PAUŠ $\check{S}^{a, *}$, M. Beneš ${ }^{a}$, J. KRATOChVÍL ${ }^{b}$ \\ ${ }^{a}$ Department of Mathematics, Faculty of Nuclear Sciences and Physical Engineering, \\ Czech Technical University in Prague, Trojanova 13, Prague, Czech Republic \\ ${ }^{b}$ Department of Physics, Faculty of Civil Engineering, \\ Czech Technical University in Prague, Thákurova 7, Prague, Czech Republic
}

\begin{abstract}
The interpretation of the experimentally determined critical distance of the screw dislocation annihilation in persistent slip bands is still an open question. We attempt to analyze this problem using the discrete dislocation dynamics simulations. Dislocations are represented by parametrically described curves. The model is based on the numerical solution of the dislocation motion law belonging to the class of curvature driven curve dynamics. We focus on the simulation of the cross-slip of one edge dislocation curve bowing out of the wall of a persistent slip band channel and one screw dislocation gliding through the channel. The dislocations move under their mutual interaction, the line tension and the applied stress. A cross-slip leads to annihilation of the dipolar parts. In the changed topology each dislocation evolves in two slip planes and the plane where cross-slip occurred. The goal of our work is to develop and test suitable mathematical and physical model of the situation. The results are subject to comparison with symmetric configuration of two screw dislocations studied in papers by Pauš et al. The simulation of the dislocation evolution and merging is performed by the improved parametric approach. Numerical stability is enhanced by the tangential redistribution of the discretization points.
\end{abstract}

DOI: $10.12693 /$ APhysPolA.128.737

PACS: 02.70.Bf, 61.72.Lk, 02.30.Jr

\section{Introduction}

Cross-slip is one of the most important single processes underlying the complex spatio-temporal developments in microstructure leading to hardening and the dislocation pattern formation [1]. Cross-slip allows the screw dislocations to change the slip planes and thus to bypass obstacles or to glide to annihilation with a screw dislocation of the opposite sign on a neighboring slip plane $[2,3]$.

In our previous works we studied the interaction and the cross-slip mechanism of two screw dislocations in a persistent slip-band (PSB) channel in a symmetric configurations (see [4-7]) and obtained critical parameters for the cross-slip mechanism, such as critical slip-plane distance $h_{c}$ and critical applied stress $\tau_{c}$ required for cross-slip to occur. In this paper we focus on the nonsymmetric problem of a screw dislocation $\Gamma_{2}$ gliding in a PSB channel which interacts with an edge dislocation $\Gamma_{1}$ bowing from a channel wall (see Fig. 1).

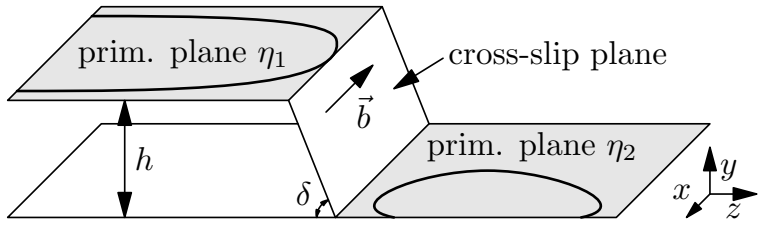

Fig. 1. Screw dislocation $\Gamma_{1}$ in primary the plane $\eta_{1}$ and the bowing edge $\Gamma_{2}$ dislocation in the primary plane $\eta_{2}$. Burgers vector is parallel with the $x$-axis.

${ }^{*}$ corresponding author; e-mail: petr.paus@fjfi.cvut.cz

\section{Model}

The key assumption is that the motion of the dislocation $\Gamma_{i}$ is governed by the law of the mean curvature flow

$$
B v_{i}=T_{i} \kappa_{i}+F_{i}, \quad i \in\{1,2\},
$$

where $v_{i}$ is the dislocation velocity in the normal direction, $\kappa_{i}$ the curvature and $F_{i}$ represents the sum of forces per unit dislocation length exerted on the curve $\Gamma_{i}$ in the normal direction, $B$ denotes the drag coefficient. The term $T_{i} \kappa_{i}$ represents the self-force expressed in the line tension approximation as the product of the line tension $T_{i}$ and the local curvature $\kappa_{i}$. The motion law (2.1) is treated parametrically - the planar curve $\Gamma_{i}(t), i \in$ $\{1,2\}$ is described by a smooth time-dependent position vector function $\boldsymbol{X}_{i}: S \times I \rightarrow \mathbb{R}^{3}$, where $S=[0,1]$ is a fixed interval for the curve parameter $u \in S$ and $I=\left[0, t_{\max }\right]$ is the time interval, $t$ means time. The curve $\Gamma_{i}(t)$ is given as $\Gamma_{i}(t)=\left\{\boldsymbol{X}_{i}(u, t), u \in S\right\}$. The motion law (2.1) with the addition of the tangential term $\alpha_{i}$ improving the numerical stability $[8,9]$ reads as

$$
\begin{aligned}
& B \partial_{t} \boldsymbol{X}_{i}=T_{i} \frac{\partial_{u u} \boldsymbol{X}_{i}}{\left|\partial_{u} \boldsymbol{X}_{i}\right|^{2}}+\alpha_{i} \frac{\partial_{u} \boldsymbol{X}_{i}}{\left|\partial_{u} \boldsymbol{X}_{i}\right|} \\
& +F_{i}\left(\boldsymbol{X}_{i}, t\right) \frac{\partial_{u} \boldsymbol{X}_{i}^{\perp}}{\left|\partial_{u} \boldsymbol{X}_{i}\right|},
\end{aligned}
$$

where $\boldsymbol{X}_{i}^{\perp}$ is a normal vector to $\boldsymbol{X}_{i}$. For the complete description see [5-7].

The forcing term $F_{i}$ consists of several parts (here $b$ denotes the magnitude of the Burgers vector):

- The force $F_{\text {appl }}=b \tau_{\text {appl }}$ caused by the applied resolved shear stress $\tau_{\text {appl }}$. This force is constant.

- The force $F_{\text {int }}=b \tau_{\text {int }}$ caused by the interaction 
stress $\tau_{\text {int }}$ between dislocations computed using the Devincre formula (see $[4,10]$ ).

- The force $F_{\text {wall }}=b \tau_{\text {wall }}$ caused by the stress $\tau_{\text {wall }}$ from PSB channel walls, in our case approximated by edge dislocation dipoles [11].

- The friction force $F_{\text {fr }}=4 \mathrm{MPa}$ is taken as the material constant [12].

The dislocation curve at the point $\boldsymbol{X}$ moves if the force term $F_{\text {eff }}=-T \kappa_{i}+F_{\text {appl }}+F_{\text {int }}+F_{\text {wall }}$ exceeds the lattice friction $F_{\text {fr }}$, i.e., Eq. (2.1) for each dislocation has the form

$$
B v=\left\{\begin{array}{c}
F_{\text {effl }}-F_{\text {fr }} \\
\quad \text { whenever } F_{\text {eff }}>F_{\text {fr }}, \\
0 \quad \text { whenever }-F_{\text {fr }} \leq F_{\text {eff }} \leq F_{\text {fr }}, \\
F_{\text {effl }}+F_{\text {fr }} \\
\quad \text { whenever } F_{\text {eff }}<-F_{\text {fr }},
\end{array}\right.
$$

The effective forcing terms $F_{\text {eff }}$ in primary and cross-slip planes are different, i.e.,

$$
F_{\text {effl }}^{\mathrm{p}}=-T \kappa+F_{\text {appl }}+F_{\text {int }}^{p}+F_{\text {wall }}
$$

for primary plane, and

$$
F_{\text {eff }}^{\mathrm{cs}}=-T \kappa+F_{\text {appl }} / 3+F_{\text {int }}^{\mathrm{cs}}+F_{\text {wall }}
$$

for cross-slip plane.

Whether the cross-slip mechanism occurs or not is determined by the cross-slip criterion (see [4]), i.e.,

- cross-slip occurs, whenever $F_{\text {appl }} / 3+F_{\text {int }}^{\text {cs }}>$ $F_{\text {appl }}+F_{\text {int }}^{p}$

- the motion in primary slip planes (primary slip) continues, whenever $F_{\text {appl }} / 3+F_{\text {int }}^{\text {cs }} \leq F_{\text {appl }}+F_{\text {int }}^{p}$.

The effective forces for the cross-slip criterion are determined in the screw parts of both dislocations.

\section{Simulation results}

The model was used in several configurations. We have selected two situations with different behavior of dislocation motion

- dislocation passing by in a channel without crossslip or annihilation,

- edge and screw dislocation annihilation in a channel.

The numerical simulations were performed for copper at room temperature using the following input parameters: magnitude of Burgers vector $\quad b=0.256 \mathrm{~nm}$ shear modulus [13]

$G=42.1 \mathrm{GPa}$

Poisson ratio

$$
\nu=0.34
$$

PSB channel width [14]

$1200 \mathrm{~nm}$

drag coefficient

$B=1.0 \times 10^{-5} \mathrm{Pas}$

friction stress [12]

cross-slip plane angle

$\tau_{\text {fr }}=4 \mathrm{MPa}$

$\lambda=71^{\circ}$

number of discretization segments $M=200$
The configuration for the dislocation passing situation is presented in Experiment 1 and in corresponding Fig. 2. The screw dislocation located in the lower primary slip plane is gliding through the PSB channel and the edge dislocation is bowing from a channel wall into the upper primary slip plane (see Fig. $2 \mathrm{~b}$ ). As the dislocations approach each other the interaction force between dislocations increases, however, the distance of primary slip planes is rather high $(40 \mathrm{~nm})$ causing the attractive force to be weaker. Therefore, the screw parts of both dislocations continue in evolution in their respective primary planes (see Fig. 2c). Further evolution makes the dislocations overlap (see Fig. 2d). One can see that the mutual interaction force changed shape of the bowing edge dislocation. This situation was not observed in the symmetric case [4].
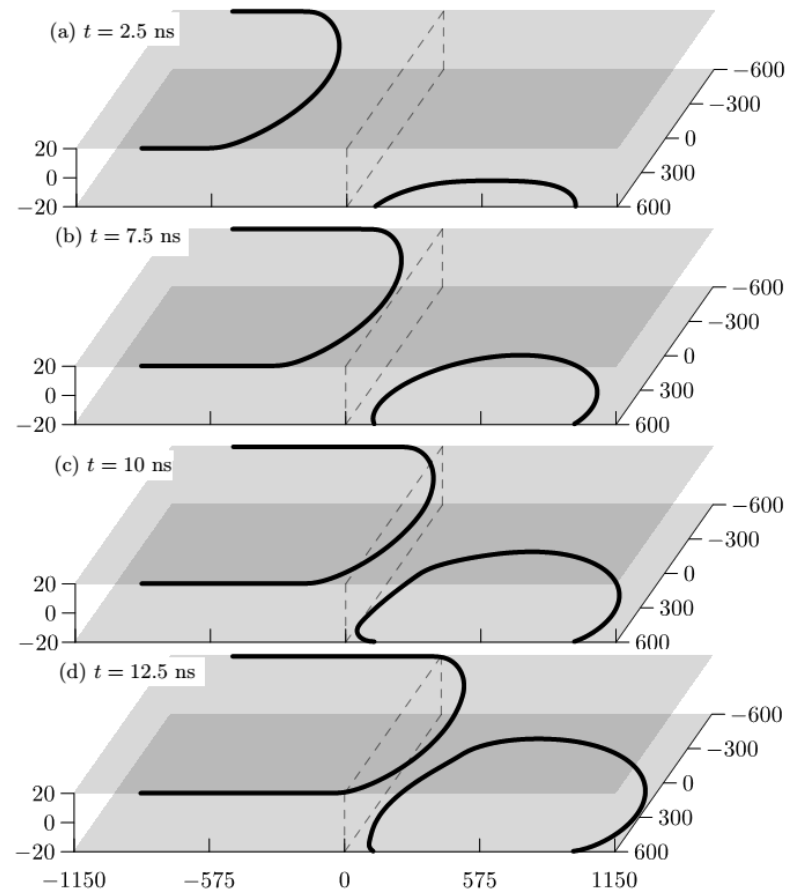

Fig. 2. Experiment 1: The edge and screw dislocation passing in a PSB channel.

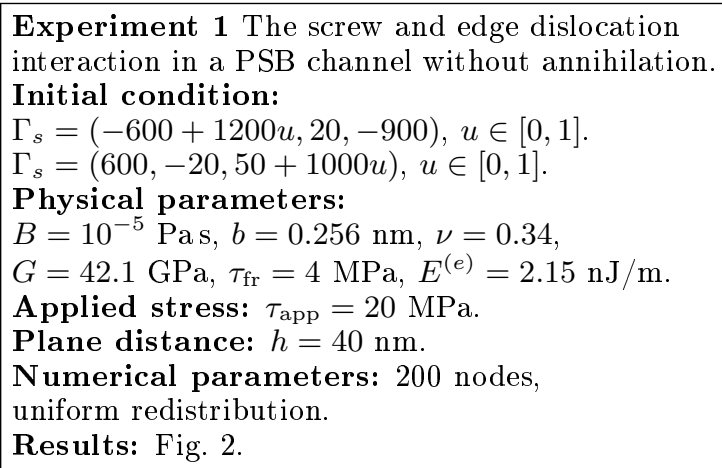

Taking the same condition as in Experiment 1 but shortening the primary plane distance $h$ to $20 \mathrm{~nm}$, i.e. Experiment 2, one can observe different behavior. Both dislocations evolve under the applied stress $\tau_{\text {app }}=20 \mathrm{MPa}$ 
(see Fig. 3a). The interaction force from the edge dislocation is slightly higher than the force produced by the screw dislocation. Therefore, at a certain time the screw tip of the gliding dislocation is forced the enter the crossslip plane but the bowing dislocation still evolves in its primary plane (see Fig. 3b and c). The screw dislocation is further attracted to the bowing dislocation and eventually annihilates in the upper primary plane (see Fig. 3d). After the annihilation there are two open dislocations slowly evolving and sticking to the channel walls (see Fig. 3e and f).

By changing the plane distance $h$ we can approximately obtain the critical plane distance for the crossslip at $\tau_{\text {app }}=20 \mathrm{MPa}$, i.e., for lower values of $h$ the cross-slip mechanism occurs while for higher values does not. This is similar to the symmetric case computations in [4]. For configuration the same as in Experiment 1 and
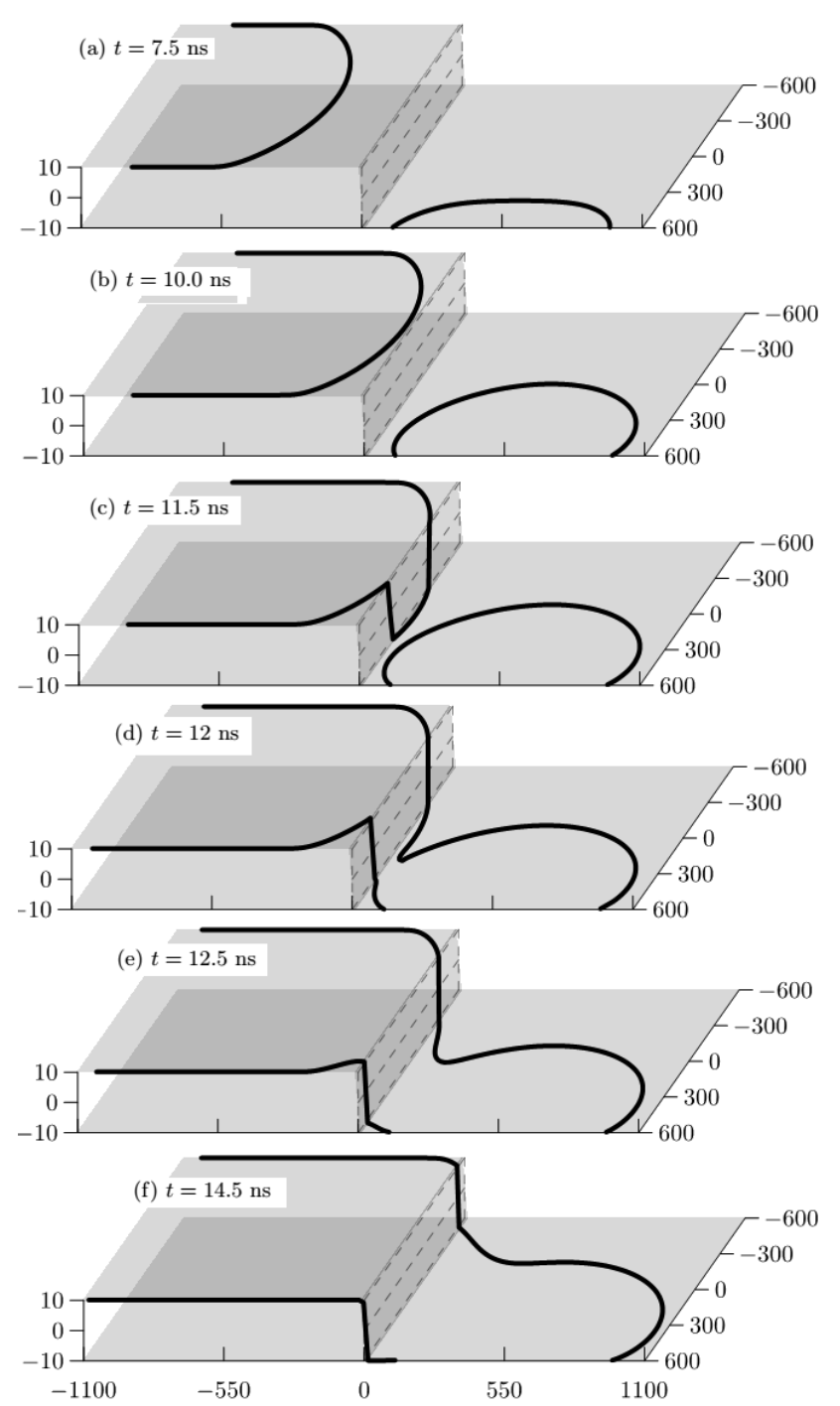

Fig. 3. Experiment 2: Edge and screw dislocations undergo the cross-slip mechanism and annihilate. Due to the scale of axes, the cross-slip plane may seem perpendicular to the primary planes, however, the angle is approximately $71^{\circ}$. $h=30 \mathrm{~nm}$, respectively $h=25 \mathrm{~nm}$, the cross-slip mechanism does not occur. Hence, the critical value $h_{c}$ lies between $20 \mathrm{~nm}$ and $25 \mathrm{~nm}$. The computations for the symmetric configuration for $\tau_{\text {app }}=21.5 \mathrm{MPa}$ provided the critical distance $h_{c}=65 \mathrm{~nm}$.

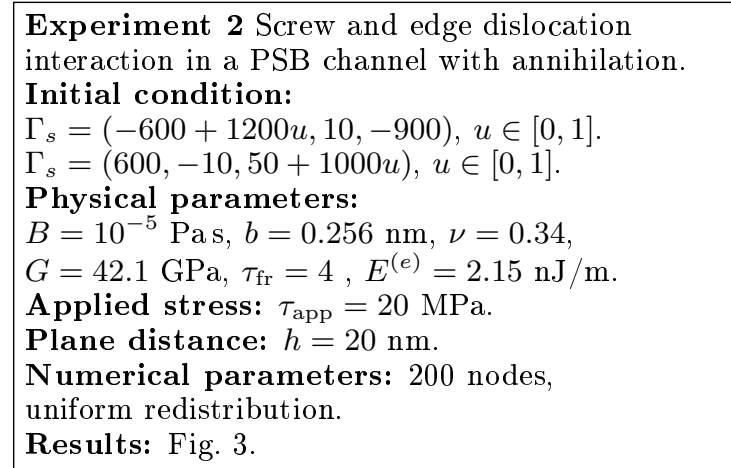

\section{Summary}

The non-symmetric configurations of dislocations in a PSB channel leading to the cross-slip mechanism and the dislocation annihilation may produce dipolar loops of the width of several $\mathrm{nm}$. Our preliminary simulations illustrate the process of the mechanism and show that the values of the critical cross-slip parameters significantly decrease, namely the critical distance of the primary planes $h_{c}$.

\section{Acknowledgments}

This work was supported by the project P108/12/1463 of the Grant Agency of the Czech Republic.

\section{References}

[1] W. Püschl, Prog. Mater. Sci. 47, 415 (2002).

[2] T. Rasmussen, T. Vegge, T. Leffers, O.B. Pedersen, K.W. Jacobsen, Philos. Mag. A 80, 1273 (2000).

[3] T. Vegge, K.W. Jacobsen, J. Phys. Condens. Matter 14, 2929 (2002).

[4] P. Pauš, J. Kratochvíl, M. Beneš, Acta Mater. 61, 7917 (2013)

[5] P. Pauš, J. Kratochvíl, M. Beneš, Philos. Mag. Lett. 94, 45 (2014).

[6] P. Pauš, M. Beneš, J. Kratochvíl, Acta Phys. Pol. A 122, 509 (2012).

[7] P. Pauš, M. Beneš, Kybernetika 45, 591 (2009).

[8] V. Minárik, M. Beneš, J. Kratochvíl, J. Appl. Phys. 107, 841 (2010).

[9] D. Ševčovič, S. Yazaki, Jpn. J. Ind. Appl. Math. 28, 413 (2011).

[10] B. Devincre, Solid State Commun. 93, 875 (1995).

[11] J. Křrišt'an, J. Kratochvíl, Philos. Mag. 87, 4593 (2007).

[12] U. Essmann, K. Differt, Mater. Sci. Eng. A 208, 56 (1996).

[13] U. Essmann, H. Mughrabi, Philos. Mag. A 40, 731 (1979).

[14] H. Mughrabi, F. Ackermann, K. Herz, in: $F a-$ tigue Mechanisms, Proc. ASTM-NBS-NSF Symp. for Testing and Materials, Kansas City 1978, p. 69. 